\title{
Underlying causes of chronic bladder dysfunction
}

\author{
Andre Marais, ${ }^{1}$ Senior lecturer and Clinical Pharmacologist, Elzbieta Osuch, ${ }^{2}$ Professor and Clinical Pharmacologist \\ 'Department of Pharmacology, School of Medicine, Faculty of Health Sciences, University of Pretoria, South Africa \\ ${ }^{2}$ Department of Pharmacology\& Therapeutics, School of Medicine, Sefako Makghato Health Sciences University, South Africa \\ Corresponding author, email:dramarais@gmail.com / andre.marais@up.ac.za
}

\section{Abstract}

General practitioners and family physicians frequently encounter patients suffering from voiding disorders. Various underlying causes such as spinal cord injury, cerebrovascular accidents, traumatic brain injury and several neurological diseases including Parkinson's disease, dementia, multiple sclerosis and vitamin deficiencies have been implicated. Chronic bladder conditions may cause social embarrassment and decrease quality of life in affected individuals. It is important to identify the most common underlying causes of bladder dysfunction and have adequate knowledge regarding the primary treatment and prevention of complications in order to reduce the economic burden created by this group of disorders.

Keywords: Chronic bladder conditions; interstitial cystitis; painful bladder syndrome; chronic urinary tract infection; urinary incontinence; hematuria; bladder cancer

\section{Introduction}

The identification and appropriate management of chronic bladder conditions remains one of the most challenging obstacles urologists, gynaecologists and general practitioners are faced with in current medical practice. Urological disorders are present from the earliest stage of human development, and continue until the end of life. ' Urological complaints mostly affect individuals' quality of life, resulting in a substantial economic impact for patients and their families, employers, healthcare funders and society at large. Bladder control problems require medical attention for several reasons. They may be indicative of certain underlying medical conditions such as diabetes, renal failure, spinal cord injuries, Parkinson's disease, dementia or multiple sclerosis. In addition, the social and psychological consequences of bladder dysfunction may be a cause of great discomfort, shame, loss of self-confidence, restriction of physical activities and withdrawal from social interactions. ${ }^{2}$

The most common chronic bladder conditions according to the Urologic Diseases in America Project include interstitial cystitis/ painful bladder syndrome, chronic urinary tract infections, urinary incontinence and hematuria. Other chronic bladder conditions less frequently encountered incorporate bladder prolapse, neurogenic bladder syndrome, bladder fistula and diverticulum, bladder enlargement (augmentation), and bladder extrophy. ${ }^{1}$ Chronic conditions of the prostate, kidney, male health, paediatrics and uncomplicated urinary tract infections are not discussed in this review.

\section{Interstitial cystitis/Painful bladder syndrome (IC/PBS)}

Interstitial cystitis and painful bladder syndrome (IC/PBS) is a debilitating heterogeneous chronic bladder disorder with multiple proposed pathophysiological mechanisms. It involves dysfunction of the urothelial barrier and associated glycosaminoglycan lining, local inflammation of the bladder and lower urinary tract, and sensory changes in the central nervous system. It is often associated with other chronic pain conditions such as chronic functional fatigue syndrome, fibromyalgia and irritable bowel syndrome. ${ }^{3-5} \mathrm{IC} / \mathrm{PBS}$ is more common in females than males (5:1) and ordinarily presents with chronic suprapubic pain, nocturia, urinary frequency and urgency. ${ }^{6}$ Diagnosis remains challenging because of overlapping symptoms with various other urological and gynecological conditions. The global incidence of IC/PBS in the general population is approximately $17.3 \%$, but shows significant variation between different countries and regions. Diagnosis involves contemporary diagnostic algorithms (duration of pelvic pain; discomfort accompanied by urinary symptoms; exclusion of other conditions by i) cystoscopy, ii) hydrodistension with bladder volume measurement, iii) bladder biopsy). ${ }^{7}$ IC/PBS has a pronounced adverse effect on psychosocial and sexual well-being. The psychological impact is often neglected by healthcare practitioners due to the lack of existing evidence in effective treatment protocols. ${ }^{8}$

Pharmacological management should be individualised for each patient in the absence of recommended guidelines. Currently patients with IC/PBS are treated symptomatically with anti-spasmodic agents, anti-constipation agents, antiinflammatory medications, tricyclic anti-depressants and botulinum toxin. ${ }^{9}$ Recent literature evidence suggests that administration of exogenous intravesical hyaluronic acid, alone or in combination with chondroitin sulphate, may be a safe and effective option for treating IC/PBS. ${ }^{10}$ Proposed future research approaches include systemic treatment with anti-nerve growth factors (elevated levels of nerve growth factors are present in bladder inflammation), ${ }^{11}$ anti-TNF alpha agents (proinflammatory cytokine release during chronic bladder urothelium inflammation), ${ }^{12} \mathrm{P} 2 \mathrm{X} 3$ receptor antagonists 
(P2X3 receptors modulate bladder activity via afferent fibers), ${ }^{13}$ and alpha1 adrenoreceptor antagonists (a receptors increase pain in chronic stress disorders). ${ }^{14}$ Preclinical studies have shown that local treatment with Toll-like receptor (TLR) antagonists may protect the host body against invading pathogens or inflammatory disorders, including autoimmune disorders and cancer. These experimental immunomodulatory drugs may provide the additional advantage of fewer systemic side effects and decrease the progression of adaptive immunity. .15

\section{Chronic urinary tract infections}

Urinary tract infections (UTIs) account for nearly $25 \%$ of all infections encountered by healthcare providers. ${ }^{16}$ UTIs are more common in females, and it is estimated that approximately $40 \%$ of all woman will experience at least one such episode during their lifetime. These infections are largely caused by uropathogenic Escherichia coli, Klebsiella pneumoniae, Proteus mirabilis, Staphylococcus $s p$ and rarely Pseudomonas or fungal elements, depending on the regional or geographical incidence pattern. Appropriate antimicrobial treatment with various agents ranging between quinolones, nitrofurantoin, fosfomycin, penicillin and sulphonamides are used as first and second line therapy in uncomplicated cases. UTIs are uncommon in males and therefore considered to be complicated if they occur. ${ }^{17}$ Complicated UTIs require specialist urological assessment to exclude structural or functional abnormalities since complications such as bacteremia, sepsis or even death may occur.

Currently more than 17 interpretations regarding recurrent (chronic) urinary tract infection (RUTI) are specified in the biomedical literature. Many features overlap, but inconsistencies regarding urinary symptoms, culture specific thresholds, bacterial species identification and number of infections per year contribute to this lack in formulating a homogeneous definition. ${ }^{18}$ Clinically, RUTIs indicate two or more infections in a six month period, or three or more infections in a year. It is important to differentiate between re-infection and relapse. Recurrent UTIs may be regarded as a re-infection if the responsible microorganism is different from the one responsible for the original infection. Considering rectal, introital, and periurethral colonization of the original responsible pathogen, re-infection may also be diagnosed if a period of at least two weeks elapse following successful antimicrobial treatment. ${ }^{19}$ These types of re-infection are regarded as uncomplicated (permitting an absence of anatomic or functional abnormalities of the urinary tract), and are not associated with long-term consequential co-morbid conditions such as hypertension or renal disease. In contrast, most physicians consider a relapsing UTI to occur within two weeks after prosperous antimicrobial treatment for the same infecting microorganism. Relapsing infections requires a more comprehensive urological evaluation, longer duration of therapy, and occasional surgical intervention. As mentioned above, the administration of exogenous intravesical hyaluronic acid markedly reduces recurrences of chronic urinary tract infections, although not currently available in South Africa. ${ }^{10}$
Worsening of chronic urinary frequency or urgency is generally associated with asymptomatic bacteriuria (ASB) in approximately $10 \%$ of pregnant woman, and $20 \%$ in woman older than 70 years by virtue of the changing female urinary microbiota. ${ }^{20}$ Elderly men have an incidence of about $6 \%$. Other special population groups with an increased susceptibility to ASB are those with immunosuppressant conditions (diabetes, HIV/AIDS, cancer), patients with catheters or spinal cord injuries, children under the age of 1 year, and those with multiple sclerosis. ${ }^{16}$ Changes in diet, previous drug or antibiotic use, surgery or urological instrumentation are additional contributing factors. Other symptoms may include nocturia, dysuria, suprapubic tenderness, costovertebral angle pain and incontinence. The absence of fever or signs of debilitating ill health demonstrates the challenges in identifying and treating this condition. It is therefore of clinical importance for the general practitioner or family physician to perform side-room urinalysis in these special population groups to prevent complicated lower urinary tract disorders and limit future hospitalizations by opportune referral to specialist urological professionals.

\section{Urinary incontinence and overactive bladder}

Urinary incontinence represents one of the most vexing chronic conditions affecting physical and social functioning. Owing to its embarrassing nature, only $25-60 \%$ of symptomatic patients seek medical care. ${ }^{21}$ It is estimated that $41 \%$ of woman over the age of 40 years and $11.2 \%$ of men above 45 years suffer from urinary incontinence. ${ }^{22,23}$ For both males and females the incidence increases with age and does not vary by race or ethnicity. Individuals residing in nursing homes show a prevalence of between $45-72 \%$ compared to those living in community settings.

The involuntary leakage of urine is classified according to the anatomical and physiological defects responsible for maintaining continence and micturition. These involve lower urinary tract, pelvic and neurological components. Functional (psychological) mechanisms are also implicated. The main types of urinary incontinence include stress, urgency and overflow incontinence, although a significant overlap between the different types often exists. ${ }^{24}$ Stress incontinence results from urethral hypermobility or intrinsic sphincteric deficiency where there is insufficient support of the pelvic floor muscles to the urethra and bladder neck, or the loss of urethral muscular tone to keep the urethra closed. It is generally associated with childbirth or direct trauma to the pelvic muscles. Any sudden increase in the intraabdominal pressure (sneezing, laughing or coughing) result in spontaneous passage of urine..$^{25}$

The terms 'urgency incontinence' and 'overactive bladder syndrome' are often used interchangeably, although the latter may or may not be associated with leaking of urine. Nocturia and frequency are the principle characteristics and occur in nearly $20 \%$ of elderly patients. Pathophysiology includes detrusor overactivity leading to uninhibited detrusor muscle contractions and the passage of small amounts of urine. Urgency incontinence may 
be secondary to neurological disorders, bladder abnormalities, or the alteration of normal bladder microflora. ${ }^{26}$

Overflow incontinence embodies the continuous leakage or dribbling of urine in the setting of incomplete bladder emptying. Bladder outlet obstruction or detrusor underactivity occurs in approximately $5-10 \%$ of elderly patients. Impaired urothelial sensory function or ineffective detrusor muscle contraction triggers symptoms similar to stress or urgency incontinence. Various etiologies of detrusor underactivity have been reported, and consist of low estrogen levels, smooth muscle fibrosis, diabetes mellitus, alcoholism, vitamin B12 deficiency and other neuropathic conditions. ${ }^{27}$ Less common causes include external masses or tumors, urethral stricture and pelvic organ prolapse.

It is important for the general practitioner to pro-actively assess and enquire about urinary continence in all patients above the age of 40 years. Initial non-pharmacological treatment of urinary incontinence includes lifestyle modifications (weight loss, dietary changes, smoking cessation, avoidance of constipation), and pelvic floor muscle (Kegel) exercises for at least 6 weeks before drug therapy should be attempted. ${ }^{28}$ Pharmacotherapy consists of antimuscarinic agents (darifenacin, oxybutynin, solifenacin, tolterodine, trospium) and beta-3-adrenergic agonists (mirabegron). The antimuscarinics are the most commonly prescribed and act by increasing bladder capacity and decreasing urgency through blockade of muscarinic acetylcholine stimulation during bladder storage. ${ }^{29}$ Therapy should be tailored according to the risks and side-effect profile of each individual drug. Mirabegron presents an alternative to patients not responding to anticholinergic therapy, where the selective stimulation of beta-3 adrenoreceptors in the detrusor muscle enhances smooth muscle relaxation.

\section{Hematuria}

Blood in the urine is a sign, and not a disease. Therapy should therefore be directed at the cause and not the symptoms. Hematuria is a common complaint encountered by approximately $4-20 \%$ of patients presenting to healthcare professionals. Urological causes for hematuria can be excluded in nearly $60 \%$ of cases, most of which often remain unidentified. In the majority of occurrences, the presence of gross hematuria may be explained by indisputable underlying conditions such as cystitis (20-25\%), urethral stones (2-20\%), anticoagulant therapy or in menstruating and postpartum women. Current best practice in the evaluation of hematuria includes urinalysis. Hematuria with unilateral flank pain may be indicative of obstructive nephrolithiasis and requires radiological imaging (radiography, ultrasound or non-contrast computed tomography). Hematuria suggestive of acute kidney injury (gross hematuria without visible clots) may indicate glomerular bleeding and requires additional cystoscopy. Microscopic hematuria as a result of menses, vigorous exercise or trauma could be excluded by history taking, followed by a repeat investigation after six weeks. Transient or persistent hematuria, especially in individuals over the age of 35 years, warrant a focused urological assessment to exclude any malignant cause. ${ }^{30}$ The presence of microscopic hematuria carries a risk of less than $1 \%$ of associated renal or upper tract tumors.

\section{Bladder Cancer}

The prevalence of bladder cancer increases with age, and is characterised by either gross or microscopic hematuria. Bladder cancer is regarded as the ninth most frequently diagnosed cancer worldwide, and rated thirteenth as the overall cause of mortality. ${ }^{31}$ It is estimated that around $12 \%$ of elderly patients presenting with hematuria may have bladder cancer. ${ }^{30}$ This high global incidence reflects the prevalence of tobacco smoking in industrialised countries. Exposure to carcinogenic chemicals used in the manufacturing of plastic and rubber (such as polyethylene or arylamine derivatives) may contribute towards developing transitional cell carcinoma (TCC) of the bladder. ${ }^{32}$ In contrast, squamous cell carcinoma (SCC) has a poor prognosis, and is essentially observed in developing African countries, where infection with endemic schistosomiasis (bilharzia) is implicated in the pathophysiology of bladder cancer. Increased urbanization, industrialization, obesity and cigarette smoking however complicate this generalization for the South African population, where an almost equal amalgamation between TCC and SCC exists. ${ }^{33}$ The 5 -year survival rate is approximately $54 \%$ in patients with TCC and $27 \%$ in those with SCC of the bladder. ${ }^{34}$

\section{Conclusion}

Chronic bladder dysfunction is associated with multiple comorbidities that have a widespread economic impact. The multifaceted heterogeneity of the underlying pathophysiology presents a challenge to the general practitioner in adequate identification and treatment of chronic bladder disorders. History and physical examination are the cornerstones of evaluation in patients with urinary tract symptoms. Complex symptomatology warrants specialist referral and investigation. This information is preeminent to formulate a long-term management plan and prognosis. Laboratory testing is necessary to diagnose and classify chronic urinary tract infections. The general practitioner should embrace every opportunity to investigate the cause of hematuria and refer all episodes not explained by an acute UTI, medication or menses for specialist urological investigation to exclude malignancy. Knowledge of the urinary microbiota, including insight into high-risk population groups, may be beneficial in the advancement of prevention, diagnosis and precision treatment of chronic lower urinary tract conditions. Being aware of this additional information may provide an opportunity to enhance clinical care without the (indiscriminant) use of antibiotics or anti-cholinergic agents.

\section{References}

1. Miller DC, Saigal CS, Litwin MS. The demographic burden of urologic diseases in America. Urol Clin North Am. 2009;36(1):11-27, v.PMC2614213. doi:10.1016/j. ucl.2008.08.004

2. Bartoli S, Aguzzi G, Tarricone R. Impact on quality of life of urinary incontinence and overactive bladder: a systematic literature review. Urology. 2010;75(3):491500. doi:10.1016/j.urology.2009.07.1325

3. Warren JW. Bladder pain syndrome/interstitial cystitis as a functional somatic syndrome. J Psychosom Res. 2014;77(6):510-5. doi:10.1016/j. jpsychores.2014.10.003 
4. Clauw DJ. Fibromyalgia and related conditions. Mayo Clinic proceedings. 2015;90(5):680-92. doi:10.1016/j.mayocp.2015.03.014

5. Gewandter JS, Chaudari J, Iwan KB, Kitt R, As-Sanie S, Bachmann G, et al. Research Design Characteristics of Published Pharmacologic Randomized Clinical Trials for Irritable Bowel Syndrome and Chronic Pelvic Pain Conditions: an Acttion Systemic Review. J Pain. 2018;10.1016/j.jpain.2018.01.007. doi:10.1016/j.jpain.2018.01.007

6. Davis NF, Gnanappiragasam S, Thornhill JA. Interstitial cystitis/painful bladder syndrome: the influence of modern diagnostic criteria on epidemiology and on Internet search activity by the public. Transl Androl Urol. 2015;4(5):506-11. PMC4708563. doi:10.3978/j.issn.2223-4683.2015.06.08

7. Van de Merwe JP, Nordling J, Bouchelouche P, Bouchelouche K, Cervigni M, Daha LK, et al. Diagnostic criteria, classification, and nomenclature for painful bladder syndrome/interstitial cystitis: an ESSIC proposal. Eur Urol. 2008;53(1):607. doi:10.1016/j.eururo.2007.09.019

8. McKernan LC, Walsh CG, Reynolds WS, Crofford LJ, Dmochowski RR, Williams DA. Psychosocial co-morbidities in Interstitial Cystitis/Bladder Pain syndrome (IC/BPS): A systematic review. Neurourol Urodyn. 2017;10.1002/nau.23421. doi:10.1002/nau.23421

9. Andersson KE, Birder L. Current Pharmacologic Approaches in Painful Bladder Research: An Update. Int Neurourol J. 2017;21(4):235-42. PMC5756823. doi:10.5213/inj.1735022.511

10. Lazzeri M, Hurle R, Casale P, Buffi N, Lughezzani G, Fiorini G, et al. Managing chronic bladder diseases with the administration of exogenous glycosaminoglycans: an update on the evidence. Ther Adv Urol. 2016;8(2):91-9. PMC4772357. doi:10.1177/1756287215621234

11. Ochodnicky P, Cruz CD, Yoshimura N, Cruz F. Neurotrophins as regulators of urinary bladder function. Nat Rev Urol. 2012;9(11):628-37. doi:10.1038/ nrurol.2012.178

12. Tracey D, Klareskog L, Sasso EH, Salfeld JG, Tak PP. Tumor necrosis factor antagonist mechanisms of action: a comprehensive review. Pharmacology \& therapeutics. 2008;117(2):244-79. doi:10.1016/j.pharmthera.2007.10.001

13. Cockayne DA, Hamilton SG, Zhu QM, Dunn PM, Zhong Y, Novakovic S, et al. Urinary bladder hyporeflexia and reduced pain-related behaviour in P2X3-deficient mice. Nature. 2000;407(6807):1011-5. doi:10.1038/35039519

14. Mailis-Gagnon A, Bennett GJ. Abnormal contralateral pain responses from an intradermal injection of phenylephrine in a subset of patients with complex regional pain syndrome (CRPS). Pain. 2004;111(3):378-84. doi:10.1016/j. pain.2004.07.019

15. Patra MC, Choi S. Recent progress in the development of Toll-like receptor (TLR) antagonists. Expert opinion on therapeutic patents. 2016;26(6):719-30. doi:10.10 80/13543776.2016.1185415

16. Foxman B. Epidemiology of urinary tract infections: incidence, morbidity, and economic costs. Am J Med. 2002;113 Suppl 1A:5s-13s.

17. Bono MJ, Reygaert WC. Urinary Tract Infection. StatPearls. Treasure Island (FL): StatPearls Publishing StatPearls Publishing LLC; 2017.

18. Malik RD, Wu YR, Zimmern PE. Definition of Recurrent Urinary Tract Infections in Women: Which One to Adopt? Female pelvic medicine \& reconstructive surgery. 2017;10.1097/spv.0000000000000509. doi:10.1097/spv.0000000000000509
19. Russo TA, Stapleton A, Wenderoth S, Hooton TM, Stamm WE. Chromosomal restriction fragment length polymorphism analysis of Escherichia coli strains causing recurrent urinary tract infections in young women. J Infect Dis. 1995;172(2):440-5.

20. Brubaker $L$, Wolfe AJ. The female urinary microbiota, urinary health and common urinary disorders. Ann Transl Med. 2017;5(2):34. PMC5300856. doi:10.21037/ atm.2016.11.62

21. Schlenk EA, Erlen JA, Dunbar-Jacob J, McDowell J, Engberg S, Sereika SM, et al. Health-related quality of life in chronic disorders: a comparison across studies using the MOS SF-36. Qual Life Res. 1998;7(1):57-65.

22. Shamliyan TA, Wyman JF, Ping R, Wilt TJ, Kane RL. Male urinary incontinence: prevalence, risk factors, and preventive interventions. Rev Urol. 2009;11(3):14565. PMC2777062.

23. Minassian VA, Yan X, Lichtenfeld MJ, Sun H, Stewart WF. The iceberg of health care utilization in women with urinary incontinence. Int Urogynecol J. 2012;23(8):1087-93. PMC3905313. doi:10.1007/s00192-012-1743-x

24. Holroyd-Leduc JM, Tannenbaum C, Thorpe KE, Straus SE. What type of urinary incontinence does this woman have? JAMA. 2008;299(12):1446-56. doi:10.1001/ jama.299.12.1446

25. Rogers RG. Clinical practice. Urinary stress incontinence in women. N Engl J Med. 2008;358(10):1029-36. doi:10.1056/NEJMcp0707023

26. Nygaard I. Clinical practice. Idiopathic urgency urinary incontinence. N Engl J Med. 2010;363(12):1156-62. doi:10.1056/NEJMcp1003849

27. Aldamanhori R, Chapple CR. Underactive bladder, detrusor underactivity, definition, symptoms, epidemiology, etiopathogenesis, and risk factors. Curr Opin Urol. 2017;27(3):293-9. doi:10.1097/mou.0000000000000381

28. Dumoulin C, Hay-Smith EJ, Mac Habee-Seguin G. Pelvic floor muscle training versus no treatment, or inactive control treatments, for urinary incontinence in women. The Cochrane database of systematic reviews. 2014;10.1002/14651858.CD005654.pub3(5):Cd005654. doi:10.1002/14651858. CD005654.pub3

29. Finney SM, Andersson KE, Gillespie Jl, Stewart LH. Antimuscarinic drugs in detrusor overactivity and the overactive bladder syndrome: motor or sensory actions? BJU Int. 2006;98(3):503-7. doi:10.1111/j.1464-410X.2006.06258.x

30. Khadra MH, Pickard RS, Charlton M, Powell PH, Neal DE. A prospective analysis of 1,930 patients with hematuria to evaluate current diagnostic practice. J Urol. 2000;163(2):524-7.

31. Antoni S, Ferlay J, Soerjomataram I, Znaor A, Jemal A, Bray F. Bladder Cancer Incidence and Mortality: A Global Overview and Recent Trends. Eur Urol 2017;71(1):96-108. doi:10.1016/j.eururo.2016.06.010

32. Chung KT. Occurrence, uses, and carcinogenicity of arylamines. Front Biosci (Elite edition). 2015;7:322-45.

33. Heyns CF, van der Merwe A. Bladder cancer in Africa. Can J Urol. 2008;15(1):3899-908.

34. Rausch S, Hofmann R, von Knobloch R. Nonbilharzial squamous cell carcinoma and transitional cell carcinoma with squamous differentiation of the lower and upper urinary tract. Urol Ann. 2012;4(1):14-8. PMC3271444. doi:10.4103/0974-7796.91615 